\title{
CUERPO PERSONALIZADO Y EDUCACIÓN FÍSICA
}

\author{
PERSONALIZED BODY AND PHYSICAL EDUCATION
}

CORPO PERSONALIZADO E EDUCAÇÃO FÍSICA

Luis Eduardo Ospina Lozano ${ }^{1}$

\section{Resumen}

\begin{abstract}
El artículo trata acerca de la importancia de replantear la visión del cuerpo en la educación física. Se inicia con una breve pero clara descripción de la problemática actual moderna y su dinámica deshumanizante aplicada, en su orden, a lo social, a lo educativo, a la educación, a la educación física y al cuerpo objeto. Seguidamente, se aborda la propuesta del cuerpo personalizado y sus generalidades filosóficas, destacándose en ello, el carácter integrador y la manifestación de la existencia incorporada para dar cuenta de la persona en su totalidad. Se finaliza con las implicaciones pedagógicas, orientadas bajo los principios personalizantes de singularidad, autonomía y apertura, implicaciones reales y susceptibles de aplicación inmediata en el ámbito escolar. Igualmente, el artículo constituye un grado de avance dentro de la investigación "Persona, educación física y desarrollo humano" que se realiza en la Facultad de Ciencias de la Universidad Libre con sede en Bogotá, Colombia.
\end{abstract}

Palabras clave: cuerpo; educación física; persona; personalización

\section{Abstract}

The paper addresses the importance of reconsidering how the body is viewed in physical education. We start with a brief but clear description of the current modern issue and its dehumanizing dynamics applied to social and educational aspects, to education, to physical education, and to the body as an object. Then, we address the proposal of the personalized body and its philosophical generalities, highlighting the integrating nature and the manifestation of incorporated existence in order to give an account of the person as a whole. We conclude with the pedagogical implications, guided by the personalizing principles of uniqueness, autonomy, and openness, which are real implications as well as immediately applicable to the school environment. The article also shows the progress made in the research "Person, physical education, and human development" carried out by the School of Science of Universidad Libre in Bogotá, Colombia.

Keywords: body; physical education; person; personalization

1 Magíster en Educación de la Universidad de la Sabana. Especialista en pedagogía de la lúdica de la Fundación Universitaria Los Libertadores. Especialista en educación ambiental de la Fundación Universitaria Los Libertadores. Licenciado en Educación Física de la Universidad Pedagógica Nacional. Docente investigador del departamento de Educación Física de la Universidad Libre. Docente de la Universidad de la Sabana. Docente de educación física del Colegio Distrital Antonio Nariño. Correo electrónico: luisospinalozano@gmail.com 


\section{Resumo}

0 artigo trata da importância de repensar a visão do corpo na educação física. Começamos com uma breve, mas clara descrição da problemática atual moderna e sua dinâmica desumanizante aplicada, nesta ordem, ao social, ao educacional, à educação, à educação física e ao corpo-objeto. Em seguida, abordamos a proposta do corpo personalizado e suas generalidades filosóficas, enfatizando nele o caráter integrador e a manifestação da existência incorporada para dar conta da pessoa em sua totalidade. Concluímos com as implicações pedagógicas, orientadas pelos princípios personalizadores da singularidade, autonomia e abertura, implicações reais e suscetíveis de aplicação imediata no ambiente escolar. Da mesma forma, o artigo constitui um avanço na pesquisa "Pessoa, Educação Física e Desenvolvimento Humano", realizada na Faculdade de Ciências da Universidade Libre, em Bogotá, Colômbia.

Palavras-chave: corpo; educação física; pessoa; personalização

Fecha de recepción: 30 de septiembre de 2016

Fecha de aprobación: 9 de julio de 2018

Para citar este artículo:

Ospina, L. (2017). Cuerpo personalizado y educación física. Lúdica Pedagógica, 26, 83-89. 


\section{PUNTO DE PARTIDA}

Hoy es evidente la pérdida del hombre, y con ello, la deshumanización del universo, pues más allá de la simple presencia física, al ser humano le corresponde hallarse como parte integrante del cosmos, esto de forma interdependiente y con fundamento autorrealizativo. Pero los actos de la humanidad apuntan a lo contrario: guerra, muerte, desolación, explotación, contaminación, etc. Relaciones, que, al decir de López (1996), tienen su origen en la manera de ver la realidad al estilo moderno, o sea, bajo el poder controlador y dominante de todo lo existente y bajo el falso concepto de autonomía enfocado en el individualismo. Justo cuando aparentemente el hombre hace uso de su capacidad típica razonativa, que, se supone, lo hace equilibrado, analítico y reflexivo para distinguir lo bueno de lo malo y encaminarse por la senda del bien, es cuando surge y se desenvuelve toda la trama deshumanizante... Es el momento en que se cambia el ser por el tener.

Preferir tener que ser es romper con la armonía universal, pues el hombre, al no encontrarse en relación estrecha con sus semejantes, con Dios y con la naturaleza, pierde dignidad y atropella lo circundante; todo cobra un precio y todo pierde valor; de este modo se pone de manifiesto la cosificación del ser humano. Se entra en una crisis existencial con claros síntomas de grave enfermedad social: actitud existencial provisional, actitud vital fatalista, ideal colectivista, fanatismo, vacío existencial (Luna, 1996).

De igual manera, falsamente se ha acentuado la creencia (también como efecto moderno) de que el proceso educativo responde al tipo de hombre y sociedad en que se contextualiza. Cuestión lógica, entonces, que, para una sociedad moderna y un hombre moderno, deba consolidarse una educación moderna. Esto equivale a decir que dicho tipo de proceso educativo se enmarca dentro de la competitividad, el control, el dominio, el ansia de poder, etc. Se trata de una antieducación, que respondiendo al primado razonativo, se preocupa más por informar que por formar. Así, los contenidos, las metodologías y los tecnicismos se han puesto a la vanguardia en el desarrollo educacional, olvidándose de los fines humanos a los cuales deben responder.
La consecuencia es desastrosa: desintegración humana y reducción del hombre a lo cognitivo. De ahí que Restrepo (1994) acuse a la escuela de ser auténtica heredera de la tradición viso-auditiva, y de realizar una labor insuficiente, al olvidar el cuerpo como factor educativo fundamental.

Con la afirmación del profesor Restrepo entramos de lleno al campo de la educación física. El marco modernista que engloba el proceso educativo trae para el hombre, en cuanto cuerpo, toda una serie de vejámenes que le truncan como persona. Al ser la escuela viso-auditiva, lo espacio-temporal, en el ser humano, al igual que lo lúdico y recreativo, pasan a un segundo plano y quedan al servicio de lo académico, lo disciplinar adaptativo o lo deportivista. Luego, el cuerpo deja de ser un elemento integrante de la persona y pierde su consolidado educativo, pues la mera exterioridad objetiva no constituye la integralidad humana.

En estas circunstancias no es de extrañar que la educación física sea vista como la cenicienta de las asignaturas escolares, y en casos extremos (que no son pocos) sea inexistente en los llamados "planteles educativos", a tal punto que Bonilla (1994) se refiere a la educación física como un área que goza de desprecio por parte de la sociedad en general. Ante dicha situación, y como mecanismos de recobro valorativo, algunos "defensores" de la educación física han optado por la teorización de la misma o la jornada "sudorativa" con claros síntomas masificadores y represivos que más parece ejercicio militar que proceso educativo.

De cualquier modo, se está fomentando la deshumanización que promueve la vida moderna, ya que teorizar en la educación física, a partir de aspectos fisiológicos, reglamentos deportivos y técnicas deportivas, dentro de lo memorístico tradicional y de forma impositiva, no es romper con el esquema moderno, sino seguirle el juego al mismo. Un tanto más hay que decir con referencia a lo sudorativo masificado, porque desconocer en el ser humano sus características fundamentales de singularidad, autonomía y apertura es sin lugar a dudas atentar contra el bien humano en lo que concierne a lo teleológico existencial. 


\section{CUERPO PERSONALIZADO}

Urge, entonces, un movimiento redimensionador en torno a la educación física. Se trata de cerrar las puertas a lo moderno y dar paso a una formación más acorde con la esencia humana que contribuya realmente a la autorrealización de quien se educa y a la consolidación de su verdadero proyecto de vida.

En ese sentido, conviene mirar el cuerpo humano como un fenómeno realmente integral e integrador, es decir, abarcante y contenido de todos los procesos de desarrollo, y en el cual se entrelazan de modo indisoluble todas las dimensiones del actuar humano. Para este propósito suena acertada la visión personalizada del cuerpo, pues se entiende que la persona como tal no es poseedora del cuerpo, sino cuerpo mismo en dinámica existencial, esto es lo que Mounier (2006) dio en llamar la espiritualidad encarnada.

El punto de partida está en el reconocimiento absoluto de la corporalidad como el primer y fundamental modo de ser y estar del hombre en el cosmos, lo que constituye una formalidad real materializada en lo sensible y cinético, que abarca elaboradas manifestaciones significativas. "En el caso del hombre, de un organismo que se apodera del mundo que le es dado conocer como real y sobre el que actúa de un modo absolutamente personal (conducta motriz)" (Cecchini, 1996, p. 53). Lógicamente, se trata de la existencia incorporada y el planteamiento hecho por Cajigal (1979) acerca del cuerpo, pero más que cuerpo. La referencia está dada desde lo real primigenio que enmarca lo espacial-temporal-causal y cuya dinámica implica la esencia de lo existencial humano.

Yendo más lejos, puede afirmarse que lo corporal constituye la persona humana y esta es impensable sin su cuerpo, ya que la presencia personal de los sujetos se hace evidente desde lo elemental en cuanto a espacio y tiempo, sensación y movimiento, con implicación directa en todas las dimensiones en que se desarrolla la vida del hombre. "Yo soy persona desde mi existencia más elemental, y, lejos de despersonalizarme, mi existencia encarnada es un factor esencial de mi fundamento personal" (Mounier, 2006, p. 47).

Ahora bien, en cuanto la persona se define como la posesión plena del propio ser, las implicaciones corporales no admiten discusión, pues dicha posesión comienza a ser realidad desde las intencionalidades no cognoscitivas y cognoscitivas del cuerpo junto a su carácter de fundamento en el mundo vital, el arte y la cultura.

Así, el cuerpo personalizado es el cuerpo propio de la educación física. Cuerpo que patentiza la presencia del hombre y manifiesta los factores personales de singularidad, autonomía y apertura. Ahora, es posible afirmar que el objeto de conocimiento de la educación física es la persona humana en cuanto corporalidad, aunque se parta de lo físico, porque lo realmente educable del ser humano radica en su integralidad y no específicamente en lo material, que a lo sumo, es adiestrable. Más allá de lo material, afirma Yarce (2014), lo verdaderamente valioso de la persona está en lo intangible, pero esto subyace ineludiblemente en lo corporal.

\section{IMPLICACIONES PEDAGÓGICAS}

Una educación física centrada en el cuerpo personalizado tiene por objeto al hombre que se forma mediante la actividad física, no a los ejercicios físicos en sí mismos (Cecchini, 1996). Entiéndase que lo educable es el ser humano, su realidad personal y no su masa muscular, por tal motivo, lo cinético corporal es medio y no fin de la educación. He aquí el asunto diferencial entre lo deportivo, lo castrense y lo educativo.

Al ser medio educativo, el aprehendizaje de destrezas, habilidades o gestos motores, al igual que el desarrollo somático, trascienden hacia lo irreductible a objeto en el ser humano: su unidad espiritual y su sentido existencial. De algún modo, dichos aprehendizajes potencian y desarrollan los mencionados aspectos autorrealizativos (lo espiritual y lo existencial) sin llegar a ser vocación en sí mismos. Puede afirmarse, entonces, que el cuerpo humano es el "lugar físico" en el que acontece la verdad del cosmos, claro, si se toma la definición de verdad como reflexión, y desde este punto de vista puede decirse que el cuerpo humano es lo más adecuado para el espíritu según un enfoque analítico-funcional (Arregui y Choza, 2002).

En cuanto a proceso de desarrollo físico, lo cinético y lo corporal se constituyen como una serie de metas o pasos jerarquizados y encadenados de un 
solo aspecto del hombre... pero nunca se refieren a la persona. De modo que, cuando el deporte persigue el rendimiento físico y la eficiencia atlética, olvidándose de la persona, ocurre que hay nulidad educativa. Igual pasa con la práctica militar cuando persigue la eficiencia beligerante a través del adiestramiento corporal. En ambos momentos se fracciona al ser humano, porque solo es visto como realidad material y nada más; los aspectos síquicos, sociales, afectivos y espirituales han sido separados y olvidados; únicamente vale la máquina (cosa) cinético-corporal que, en caso de sufrir daño, puede ser remplazada fácilmente por otra, de ahí que la pérdida de vidas humanas de forma violenta y la exclusión masificada de trabajadores del campo laboral sean fenómenos comunes y cotidianos dentro de una "normal" insensibilidad.

Entre tanto, en una dinámica de cuerpo personalizado pasa algo muy diferente. El hombre se educa mientras se mueve y se mueve para educarse, por tal razón, la actividad física y la tecnología cinética están al servicio del hombre y no al contrario. Entonces, mediante lo cinético-corporal, es posible el desarrollo integral, y a su vez puede darse el reconocimiento de capacidades y limitaciones propias en múltiples aspectos de la pluridimensionalidad humana. Del mismo modo, se tiene en cuenta la dimensión expresivo-comunicativa de la corporalidad y sus intencionalidades frente a lo cognoscitivo y lo social, porque el cuerpo no solo hace parte del yo, sino que lo expresa. En la corporalidad residen, de manera física (material, si se quiere), tanto la intimidad como la primera y más profunda comunicación.

La singularidad, como característica fundamental de la persona, más allá de la mera separación real y numérica, está conformada por todos los elementos que diferencian a cada ser humano de sus semejantes y tienen que ver exclusivamente con su actuación, pensamiento y relación con el medio que, de forma indiscutible, le dan una identidad que se hace irrepetible e irremplazable, básica para llevar a cabo el proceso autorrealizativo (García, 1988). Luego, una educación física que tiene por "objeto" el cuerpo personalizado evita a toda costa las prácticas masificadoras y represivas, reconoce en el ser personal su principio de singularidad y atiende de la manera más adecuada las diferencias individuales en cada aspecto (culturales, afectivas, de interés, de ritmo, etc.). Tam- bién fomenta la actividad creativa y original, desde el hacer mismo, hasta lo existencial, humanamente hablando.

La didáctica, entonces, debe estar más enmarcada dentro del equilibrio racional-afectivo que inclinada hacia lo vertical razonativo en donde la "autoridad" se da por imposición. Es decir, que todo lo que atañe al proceso de enseñanza-aprendizaje está implícito dentro del reconocimiento de las capacidades que evidencian la excelencia personal. Tratándose del maestro, se verifica la habilidad motivadora y sensible que, de alguna manera, representa el aliciente más favorable para el aprendizaje fuera de lo adversivamente inhumano. Es, en concreto, la muestra clara del poder legítimo que media en una relación educativa interpersonal.

"Ser persona y ser libre son conceptos inseparables. Uno no existe primero y después es libre. Existir como persona es al mismo tiempo ser libre. Esta libertad es tan esencial en la persona como su ser-enel-mundo y su ser-con-otros" (Vásquez, 1990, p. 23). Con esto vale decir que el respeto al carácter autónomo del ser humano es también constante dentro de la práctica de una educación física que cree en el cuerpo personalizado. Es normal que las actuaciones de todos los involucrados en el proceso educativo estén matizadas por frecuentes posibilidades de ejercicio libre y comprometido, díganse situaciones que permiten la elección y la decisión, con la consabida asunción de las consecuencias.

Una educación física que tiene en cuenta el cuerpo personalizado permite situaciones de encuentro mutuo humano entre sus integrantes y también estimula las buenas relaciones de estos con la naturaleza y con el ser supremo; porque, a ciencia cierta, el hombre solo llega a ser persona en la medida en que pone a prueba su principio de apertura en el communicare, o sea, el trato por medio del cual se halla a sí mismo en relación dialéctica con todos y con todo lo circundante.

Como condición número uno de desarrollo humano, la educación física que tiene como eje fundamental el cuerpo personalizado, encamina todos sus esfuerzos para que cada sujeto llegue a consolidar la capacidad de gestarse y llevar a cabo su propio proyecto de vida, pues es verídico que cada quien encuentra 
su autorrealización por sentido vocacional que nunca se repite entre las personas, es decir, que es único e irremplazable. "[...] la persona no es un concepto, una idea; la persona es indefinible porque no es una cosa, un objeto; la persona es un PROYECTO" (Peralta, 1994, p. 3).

La integridad personal permite hablar del hombre en cuanto a dimensionalidad, características propias de la persona y actuaciones humanas sin pretender separaciones o sumatorias, más bien afirmando la condición infragmentada del ser personal y su complejidad en la interrelación de dichos elementos. Gómez y Bohórquez (1996), haciendo alusión a la persona como la forma más propia y única del ser hombre, fundamentada en la unidad, hacen referencia al todo indisoluble, porque es posible tratar de las dimensiones, características y actuaciones humanas por separado, pero no de la persona en la misma situación. La persona solo es tal en su unidad antropológica e impensable en la disociación, aspecto vital dentro de una educación física que tiene como eje el cuerpo personalizado.

Fuera de lo enunciado hasta el momento, si se tiene en cuenta que la calidad de la educación está representada por el conjunto de propiedades que permiten mejorar su apreciación y le son inherentes, una educación física centrada en el cuerpo personalizado propugna por horarios, elementos y espacios adecuados a la formación del hombre. Asimismo, permite relaciones enmarcadas dentro de lo humano: afectividad, comunicación, deliberación, propuestas y métodos que implican el respeto de lo natural en la persona, lo cual exige el conocimiento a cabalidad del proceso de desarrollo físico, psíquico, social y su dinámica espiritual.

\section{CONCLUSIONES}

En González (2004) encontramos que los fenómenos de precisión, eficacia y competitividad, han señalado un derrotero despersonalizante para la sociedad de hoy, en donde se manifiestan la pérdida de la identidad por el código y la primacía de los hechos sobre las ideas. Así, se plantea una necesaria y urgente transformación en términos de humanización; es un llamado al protagonismo del hombre para un desarrollo social más justo. Sin lugar a dudas, y teniendo en cuenta que no hay cambio social que no pase por lo educativo, se consolida la educación física como uno de los elementos fundamentales en la necesidad de cambio. En ese sentido, y teniendo en cuenta que el cuerpo es el factor principal de mediación, urge también un cambio en la concepción y tratamiento de lo corporal en la educación física; horizonte que se vislumbra claro en la personalización del cuerpo.

Aparte de lo ya dicho, podemos sintetizar que, en cuanto hay proceso educativo, en la propuesta del cuerpo personalizado lo importante no es el aprehendizaje de "maromas", sino el grado de formación humana que se puede llegar a generar y desarrollar desde la práctica de dichos eventos. Luego, la actividad física como tal viene a ser medio y no fin de la formación humana. Significa esto que el cuerpo personalizado es una totalidad de existencia y perfección y no una simple arista de ser humano sin sentido alguno más allá del materialismo. Esto también significa que la educación física se orquesta en la armonía pura de la educación, y que, cuando busca cumplir con sus obligaciones educacionales, debe enseñar algo más que habilidades técnicas, aptitud física, arte y pericia en el deporte, hábitos saludables, reglas y estrategias; debe encontrar su razón de ser en el contexto de la educación en general (Peralta, 1994).

En últimas, la propuesta del cuerpo personalizado en la educación física es una cuestión que puede ser realidad si se gesta en primera medida por quienes están en contacto directo con el ramo, es decir, los y las licenciados(as) en educación física. La invitación es llegar a establecer y cumplir el compromiso que corresponde dentro de lo personal y social. Es un cambio de actitud que se inscribe dentro de la reflexión pedagógica diaria e incita, en la construcción de un verdadero ambiente educativo, a la transformación de prácticas que van desde lo metodológico y didáctico hasta la formulación y consecución de fines, todo respondiendo al proceso de perfección humana.

\section{REFERENCIAS}

Arregui, V., y Choza, J. (2002). Filosofía del hombre. Una antropología de la intimidad. Madrid: Rialp.

Bonilla, C. (1994). Pedagogía y cultura física. Armenia, Colombia: Kinesis. 
Cajigal, J. (1979). Cultura intelectual y cultura física. Buenos Aires: Kapeluz.

Cecchini, J. (1996). Personalización en la educación física. Tratado de educación personalizada (tomo 19). Madrid: Rialp.

García, H. (1988). Educación personalizada. Madrid: Rialp.

Gómez, P., y Bohórquez, M. (1996). Enfoque personalizador en la educación. Actualidad educativa, 13, 24-34.

González, J. (2004). Personalización. Madrid: Prentice Hall.

López, M. (1996). Filosofía, modernidad y posmodernidad. Buenos Aires: Biblos.

Luna, J. (1996). Logoterapia. Un enfoque humanista existencial. Bogotá: San Pablo.
Mounier, E. (2006). El personalismo. Bogotá: Búho.

Peralta, H. (1994). Proyecto Hecper. Investigación científica en el deporte. Bogotá: Ministerio de Educación Nacional.

Restrepo, L. (1994). El derecho a la ternura. Bogotá: Arango.

Vásquez, C. (1990). Educación personalizada. Una propuesta educativa para América Latina. Bogotá: Indo-American Press Service.

Yarce, J. (2014). Ética personal en acción. Chía, Colombia: Universidad de la Sabana. 
